\title{
Dieter Fuchs
}

\section{Menippeisches Schreiben als Oszillation zwischen Struktur und Antistruktur: James Joyces Ulysses und Finnegans Wake}

Das literarische Phänomen der Menippea ist außerhalb eines kleinen Expertenkreises immer noch so gut wie unbekannt. Obwohl Mikhail Bakhtin (1984) und Northrop Frye (1957) das Verständnis dieser literarischen Form im 20. Jahrhundert revolutionierten, scheint Fryes Beobachtung aus dem Jahr 1957 auch für die Gegenwart zutreffend. Als er 1957 die literarische Form der Menippea in seiner Anatomy of Criticism zu erläutern suchte, bemerkte er, »there was not one in a thousand university English teachers [...] who knew what Menippean satire was: now there must be two or three.« (Zitiert nach Weinbrot 2005, 11)

Obwohl das menippeische Textkorpus im Kanon der Weltliteratur bestens etabliert und bekannt ist, sorgt die Tradition menippeischen Schreibens noch immer für Verwirrung. Jeder kennt Seneca, Petronius, Lukian, Apuleius, Thomas Morus, Erasmus von Rotterdam, Rabelais, Grimmelshausen, Dryden, Sterne, Swift, Cyrano de Bergerac, Voltaire, Byron, James Joyce, Thomas Mann und Flann O’Brien. So gut wie jeder hat jedoch Schwierigkeiten, deren Texte hinsichtlich der beinahe vergessenen Tradition menippeischen Schreibens einzuordnen. Man kennt also viele Texte des menippeischen Korpus und weiß sie in Rekurs auf die Gattungstraditionen des mock-heroischen Epos, der Romanze, des alten oder neuen Romans mehr schlecht als recht zu lesen; so gut wie niemand weiß jedoch mit diesen Texten in Rekurs auf den Deutungshorizont der menippeischen Satire etwas anzufangen. Dieses Phänomen resultiert insbesondere aus der Tatsache, dass das menippeische Korpus Texte umfasst, die unter anderem deshalb als notorisch schwer und unlesbar gelten, weil sie die traditionelle Leseerwartung eines in sich geschlossenen, sinnzentrierten plots konsequent verweigern. Statt Antworten $\mathrm{zu}$ geben, werfen diese Texte in selbstreflexiver Weise unbeantwortbare Fragen auf und frustrieren als "Dialektik von Form und Selbstbezug" (Simon 2016, 72) gezielt die Erwartung ihrer Leser. Daher reflektiert die Tradition menippeischen Schreibens ein Zentralproblem einer Theorie der Prosa:

Soll die Erzählung zu ihrem Ziel kommen, dann wäre eine tiefgreifende Ambiguierung des Erzählers oder eine Dekonstruktion des Adressaten für dieses Ziel hinderlich. Die poetische Funktion als solche betreibt aber genau diese Ambiguierung, indem sie alle prag-

Dieter Fuchs, Wien

Ә Open Access. (C) 2021 Dieter Fuchs, publiziert von De Gruyter. (cc) BY Dieses Werk ist lizenziert unter einer Creative Commons Namensnennung 4.0 International Lizenz. https://doi.org/10.1515/9783110731569-017 
matisch gerichteten Bezugnahmen selbstbezüglich werden lässt und somit die Positionen der Kommunikation auffächert, statt sie funktionieren zu lassen. [...] Form und Selbstbezug profitieren voneinander, aber sobald sich der Selbstbezug maximieren will, ist der bei der Gestalt verharrende Eigenwille der Form dabei hinderlich. Genau diese Dialektik ist es, die es notwendig macht, über die Form hinausgehen zu müssen, wenn das Ziel der ästhetischen Durcharbeitung des Materials darin besteht, ein Maximum an Selbstbezug erreichen zu wollen. `Nach der Form ^ meint also diejenige ästhetische Realität, die Form im Wissen um deren Limitiertheit übersteigt, um in den Bereich zu kommen, in dem die immanente Stoppregel der Form nicht mehr gilt.

(Simon 2016, 71-72)

Die Selbstreflexivität menippeischer Texte wird durch ein Arsenal experimenteller Verfahren - Bakhtin (1984, 114-119) abstrahiert vierzehn menippeische Textbausteine - realisiert, deren detaillierte Erörterung den Rahmen eines Aufsatzes sprengen würde. Man kann jedoch zusammenfassend feststellen, dass diese Verfahren dazu beitragen, die traditionelle Koordinierung des Hier und Jetzt aufzubrechen, überkommene Wissenssysteme, Weltbilder und deren philosophisch-literarische Modellierung in Frage zu stellen. Da die menippeischen Textbausteine traditionelle Repräsentationsmuster gezielt aufrufen, um sie zu parodieren bzw. zu demontieren, ist die experimentelle Radikalität menippeischen Schreibens als hochgradig autoreferentielles Verfahren im Spannungsfeld antizipierter Schreib- und Denkstrukturen vs. diese Formen sprengender Anarchie fassbar. Die um dieses Phänomen kreisenden Überlegungen meines Beitrags strukturieren sich wie folgt.

Obwohl das Hauptaugenmerk darauf abzielt, James Joyce als Repräsentanten menippeischen Schreibens vorzustellen und sowohl dessen Ulysses als auch Finnegans Wake als menippeische Texturen zu erörtern, scheint es zunächst wichtig, die Tradition menippeischen Schreibens zu beleuchten. Daher ist in einem ersten Schritt zu zeigen, welche Verständnishürden dafür verantwortlich sind, dass die menippeische Tradition in der Literatur- und Kulturwissenschaft ein marginalisiertes und weitgehend rätselhaftes Feld geblieben ist. In einem zweiten Schritt soll die Menippea einerseits hinsichtlich ihrer Geschichte als historische Gattung, andererseits in Rekurs auf ihren weltanschaulichen Kern als ahistorische Schreibweise erfasst werden. Vor diesem erkenntnistheoretischen Hintergrund ist dann in einem dritten Schritt zu zeigen, wie Joyce in Ulysses und Finnegans Wake die menippeische Tradition umsetzt, um die Literatur des zwanzigsten Jahrhunderts zu revolutionieren. In diesem Zusammenhang ist der Fokus weniger auf die sprichwörtlich gewordene experimentelle Radikalität Joyce'schen Schreibens zu richten, die Ulysses und Finnegans Wake zu notorisch unverständlichen und potentiell unlesbaren Texturen machen, wie die Mehrzahl der Lesenden behauptet. Statt wohlbekannte Aspekte der formsprengenden Komplexität zu wiederholen, ist zu zeigen, dass Ulysses und Finnegans Wake aller experimenteller Verfahren zum Trotz nicht nur eine menippeisch-anarchische >Antistruktur aufweisen, sondern diese in ästhetischer Spannung zu sehr traditionellen Strukturen und Schreibmustern modellie- 
ren. Diese »Dialektik von Form und Selbstbezug« (Simon 2016, 72) gilt es als Ausblick auf die Theorie der Prosa abschließend zu reflektieren.

\section{Verständnishürden menippeischen Schreibens}

Verlorene Urtexte: Obwohl Menippos von Gadara und Marcus Terentius Varro als Begründer einer hellenistischen und römischen Tradition menippeischen Schreibens bekannt sind, sind deren Texte verschollen und allenfalls fragmentarisch erhalten. Wir können uns aufgrund dieser Überlieferungslage über die Urtexte der menippeisch-varronischen Tradition also so gut wie kein Bild machen. Erschwerend kommt zu dieser Problematik hinzu, dass Lukian in einigen seiner Texte den menippeischen Gattungsgründer >Menippos` als literarische Sprecherfigur auftreten lässt, was unter anderem dazu führte, dass der Altphilologe Rudolf Helm (1967 [1906]) den historischen Menipp aus den höchst fantastischen Texten Lukians heraus zu erschließen suchte.

Gattungspoetische Problematik: Aufgrund der interkulturellen Heterogenität menippeischen Schreibens ist die menippeische Tradition nur sehr bedingt in poetologische Raster einzuordnen. Während die auf Menippos und Lukian zurückgehende hellenistische Tradition wegen ihrer unklassischen Regellosigkeit einen Alptraum für die Regelpoetik darstellt, ist die von Varro über Seneca reichende römische Tradition poetologisch besser klassifizierbar. Als Konsequenz hat man versucht, die hellenistisch-menippeische Regellosigkeit von der römisch-varronischen Regelhaftigkeit abzugrenzen und daraus die poetologisch ıbereinigte، Gattung der varronischen Satire zu extrapolieren. Wie die von Helmut Castrop (1983) verfasste Monographie über die varronische Satire als unintendierte Leerstelle allerdings zeigt, gibt es ein großes Korpus regelpoetisch-theoretischer Abhandlungen über die varronische Satire, das jedoch mit einem ebenso großen Defizit praktischer Lektüreeinsicht hinsichtlich der Unterscheidbarkeit von menippeischer und varronischer Satire einhergeht.

Satirebegriff: Sehr eng mit dieser Problematik verbunden ist die irreführende Bezeichnung der menippeischen Tradition als menippeische Satire. Der regelpoetisch überbesetzte Begriff der Satire bzw. des Satirischen als Geißelung lebensweltlicher Fehler und Laster verstellt die Leseerwartung eines menippeischen Textes. Die Leser^in nimmt intuitiv an, bei der Menippea handle es sich um Satire im Sinne Horazens oder Juvenals, nicht jedoch im Sinne Quintilians, der satura nicht als topische Satire, sondern als heterogenes Potpourri definiert (Quintilianus 1970, 10.I.9395). Im Gegensatz zur römischen Satire Horazens und Juvenals handelt es sich bei der Menippea nicht um topische, sondern um anthropologisch-philosophische Satire, also um kynisch-sokratische Menschheits-Satire, die sich grundlegend vom 
römischen Begriff des Satirischen unterscheidet. Die menippeische Satire geißelt keine Fehler und Laster, sondern stellt die menschliche Natur und deren Erkenntnisfähigkeit an sich in Frage.

Die menippeische Satire als romanähnliche Erzählprosa: Ein weiteres Verständnisproblem menippeischen Schreibens geht auf die irrtümliche Gleichsetzung der menippeischen Tradition mit romanähnlicher Erzählprosa einher. Wenn etwa Werner von Koppenfels die Menippea als »Vor- und Konkurrenzform des bürgerlichen Romans« $(1981,17)$ bezeichnet, so impliziert diese Wertung teleologisch-zielgerichtetes Erzählen, das im bürgerlichen Roman des achtzehnten Jahrhunderts als Entwicklungsszenario entfaltet wird. Dies impliziert die irrtümliche Leseerwartung, menippeisches Erzählen beschreibe im aristotelischen Sinne die fortschrittszentrierte Entwicklung eines epischen Handlungsstrangs, dessen Ende sich als telos - also als logisch-zielgerichtete Konsequenz der Handlungskonstellation des Anfangs - ergibt.

Auch wenn der alte hellenistische Liebesroman und die Romanze einen wesentlich offeneren, seriell anstatt logisch strukturierten Handlungsbogen und fantastische anstatt realistische Geschichten mit entwicklungslosen Figuren erzählen, so weisen auch diese literarischen Formen mit der abschließenden Wiedervereinigung der zu Beginn getrennten Liebenden nach deren odysseehafter Queste durch die ganze Welt eine Art aristotelisch-teleologischen Plot auf. Nicht jedoch die Menippea.

Dass die Menippea diese Art zielgerichtet-sinnzentrierten Erzählens zunächst als narratologischen Erwartungshorizont aufruft, dessen Einlösung aber autoreferentiell und konsequent verweigert, macht sie für viele Leser^innen zum enigmatischfrustrierenden Antinarrativ. Dabei erweist sich jedoch genau diese scheinbar absurd-sinnlose Frustrationserfahrung als einsichtsfördernd, wenn man in Rekurs auf Bakhtin die Verwurzelung der menippeischen Tradition im sokratischen Dialog und in der Erkenntniskritik der sokratischen Philosophie berücksichtigt (1984, 102-112). Denn wie der sokratische Dialog endet auch jedes menippeische Narrativ in einer Aporie, d.h. in der tieferen Erkenntnis, dass man weiß, dass man nichts weiß, was durchaus erkenntnisfördernd sein kann.

Die Menippea als Gattungshybrid: Letztlich erweist es sich für die Erfassung menippeischen Schreibens als erschwerend, dass die Menippea nicht nur formale Ähnlichkeiten mit der uns bekannten Erzählprosa aufweist, sondern auch Überschneidungen mit der Gattung des Dramas, des Epos und der Lyrik. Einerseits gestaltet Lukian viele seiner Texte dramenähnlich in Dialogform, andererseits zeichnet sich die ansonsten meist in Prosa gehaltene Tradition menippeischen Erzählens durch das Prosimetrum aus: der Lyrik oder dem heroischen Epos entnommene Verseinschübe in den Erzähltext, die den ironischen Kontrast zwischen den übermenschlichen Helden des Mythos und der allzu menschlichen Trivialität 
der alltäglichen Lebenswelt erfassbar machen. Betrachtet man das menippeische Verfahren des Prosimetrums aus der Warte der Prosatheorie, so ist dieses nicht nur intertextuell, sondern auch formal selbstreflexiv, da es zeigt, dass nicht nur die Lyrik, sondern auch »jede Prosarede metrische Strukturen kennt, welche aus der Kombination von syntaktischer Gliederung, Atemzäsur, Körperrhythmik und Denkbewegung resultieren« (Simon 2013, 13, FN 14).

\section{Die Menippea als historische Gattung oder als ahistorische Schreibweise?}

Aufgrund des bisher Gesagten ergibt sich die Frage, ob die menippeische Satire als historisch gewachsene - aber regelpoetisch prekäre - Gattung zu beschreiben sei oder ob sie als ahistorische Schreibweise betrachtet werden soll. Man kann die Menippea insofern als ahistorische Schreibweise begreifen, als sie eine Anzahl klar erkennbarer Textbausteine enthält, die unabhängig von Untergattung und Epoche zeitlose weltanschauliche Konstanten menippeischen Schreibens modellieren. Bakhtin hat, wie eingangs bereits erwähnt, vierzehn solcher Textbausteine identifiziert; in meiner Forschung habe ich versucht, diesen Katalog auf eine noch überschaubarere Anzahl einzudampfen, indem ich die Menippea als Sprachrohr der Philosophie des Sokrates und deren Radikalisierung durch die Kyniker verstehe. Gleichzeitig habe ich im Dialog mit Mikhail Bakhtin, Northrop Frye und Umberto Eco (1982) versucht, die Menippea in drei historisch gewachsene Gattungstraditionen zu gliedern. Diese drei Entwicklungslinien lassen sich einerseits durch historische und kulturelle Differenz voneinander abgrenzen, andererseits zeichnen sie sich aber durch die ahistorische Gemeinsamkeit ihres weltanschaulichen Wesenskerns aus.

Die Menippea als ahistorische Schreibweise: Versucht man, die Menippea als ahistorische Schreibweise $\mathrm{zu}$ erfassen, scheint es sinnvoll, sich auf ihren philosophisch-weltanschaulichen Kern zu konzentrieren. Bekanntermaßen war Menippos von Gadara als Begründer menippeischen Schreibens ein kynischer Wanderphilosoph, der sich als Nachfolger des Diogenes von Sinope verstand. Diogenes wiederum sah sich als Nachfolger des Antisthenes, der die kynische Bewegung als Schüler des Sokrates begründet hatte. Da Diogenes die Philosophie des Sokrates in radikalerer - d. h. existentiell zugespitzterer - Weise umzusetzen versuchte als der Antisthenes-Mitschüler Plato, leitete er die Bezeichnung der von ihm begründeten Bewegung vom altgriechischen Wort des Hundes ab, um die Kreatürlichkeit der menschlichen Existenz zu betonen und das menschli- 
che Selbstverständnis als rationales Wesen in Frage zu stellen. Der Begriff Kynismus bedeutet ja bekanntlich nichts anderes als Hundephilosophie.

Sokrates führt durch den Satz sich weiß, dass ich nichts weiß vor, dass bei systematisch-radikaler Hinterfragung gängiger Wahrheiten all das, was die Schulphilosophie als swahr s erachtet, in eine Aporie - also in eine nicht mehr beantwortbare »letzte Frage« (Bakhtin 1984, 119) - mündet. Die kynischen Nachfolger des Sokrates radikalisieren diese Erkenntniskritik, indem sie alle gängigen Konzepte buchstäblich vom Kopf auf die Füße stellen und somit in ihr spara-doxes - also gegen die gängige Meinung gerichtetes - Gegenteil umkehren und durch das Bild des Hundes die menschliche Natur als kreatürlich-unwissende Existenz ausstellen.

Aus dieser Warte radikalisierten sokratischen Philosophierens verwundert es nicht, dass Diogenes von Sinope - der Schüler des Sokrates-Schülers und ersten Kynikers Antisthenes - als "verrückter Sokrates« in die Geistesgeschichte einging (Diogenes Laertios 1925, VI.54). Und da sich der Begründer menippeischen Schreibens, Menippos von Gadara, als Schüler des Diogenes inszenierte, verwundert es ebenso wenig, dass ihn Joel C. Relihan als »noch verrückteren Sokrates« bezeichnet: »If, as Plato is reported as saying, Diogenes the Cynic is a mad Socrates, we may say that Menippus is a madder Socrates." (Relihan 1989, 59; 1993, 44)

In Rekurs auf Relihans Beobachtung und Bakhtins menippeische Archäologie des sokratischen Dialogs bin ich zu dem Schluss gekommen, dass menippeisches Schreiben das sokratisch-kynische Erkenntnisparadox der menschlichen Natur inszeniert und auf diese Weise die traditionelle Leseerwartung teleologischsinnzentrierter Lektüre als Einsicht des Nichtwissens dekonstruiert. Daher findet sich meiner Forschung zufolge in jedem menippeischen Text eine parodistisch gebrochene Sokratesfigur, die nach Joel C. Relihan (1992) jeweils als Sprecher eines pseudo-platonischen Symposiums fassbar ist.

Neben ironisch gebrochenen Sokrates-Figuren und intertextuellen Verweisen auf pseudo-platonische Symposia sind als weitere ahistorische Textbausteine die Parodie der Unterweltsreise des homerischen Odysseus sowie ironisch gebrochene Odysseus- und Herakles-Figuren greifbar. Während der historische Sokrates als philosophische Inspiration der kynischen Philosophie fungiert, erweisen sich Odysseus und Herakles als Dulder und Überwinder eines unmenschlichen Schicksals mit dem kynischen Ideal des trotzig gegen die altgriechische Schicksalssemantik ankämpfenden Underdogs kompatibel. In Rekurs auf Odysseus scheint zudem relevant, dass die Zyklopenhöhle ebenso wie dessen Unterweltsreise als Archetyp dystopisch geschlossener Innenräume fungiert (Koppenfels 1981, 34) und die homerische Odyssee Modellcharakter für den hellenistischen Liebesroman aufweist - einer Gattung der Erzählprosa, mit der die menippeischen Satiriker Petronius, Apuleius und Lukian intensiv experimentieren. Nach Joel C. Relihan gestalten sich diese Gemeinsamkeiten wie folgt: 
[R]omance and Menippean satire have similar origins, and their histories touch at a number of points. The Odyssey is for both genres a thematic starting point, whether as the wanderings that precede the reuniting of lovers and families or as the fantastic adventures of a narrator whose most practiced art is that of lying.

(Relihan 1993, 179)

Die Menippea als historische Gattung; unterschiedliche Entwicklungslinien menippeischen Schreibens: Nähert man sich der Menippea als historisch gewachsener Gattung, ergeben sich drei unterschiedliche Entwicklungslinien: erstens, die lukianisch-hellenistische Tradition der menippeisch-anthropologischen Satire; zweitens, die römisch-enzyklopädistische Tradition der varronischen Satire; und drittens, die spätantik-frühmittelalterliche Summa.

Lukianisch-hellenistische Tradition der menippeisch-anthropologischen Satire: Wie bereits ausgeführt, reflektieren Lukians Werke das sokratische Wissensparadox und radikalisieren es als kynische Diatribe, die die gängigen Meinungen >paradox ‘ auf den Kopf stellt, essentialistische Antworten verweigert und stattdessen neue Fragen aufwirft. Da es im sokratischen Sinne keine fixen Wahrheiten gibt, bedeutet dies in radikaler Zuspitzung, dass sich Wahrheit und Lüge nicht essentiell, sondern allenfalls graduell unterscheiden und dass die menschliche Spezies mangels der Erkenntnisfähigkeit des Wahren nicht wirklich rational bestimmt agiert.

Römisch-enzyklopädistische Tradition der varronischen Satire: Als vir Romanorum eruditissimus bekannter Universalgelehrter (Quintilianus 1970, 10. I.95) verknüpft Varro als Begründer der römischen Entwicklungslinie menippeischen Schreibens die hellenistische Form der menippeisch-sokratischen Wissenssatire mit der römischen Tradition der topischen Satire, von der Quintilian behauptet: "satura enim tota nostra est « (1970, 10.I.93). Als Hommage an die sokratisch-hellenistische Form präsentiert Varro jedoch Sprecherfiguren, die sich als pedantische Stubengelehrte - oder, wie Northrop Frye sagen würde, als philosophi gloriosi (Frye 1957, 309) - in widersprüchliche Positionen bzw. unnützes Wissen der Schulphilosophie verstricken. Auf diese Weise warnt Varro selbstironisch vor den Auswüchsen zu umfassender Detailgelehrsamkeit. In je mehr irrelevantes Detailwissen sich der Mensch (durchaus im Sinne von Zettel's Traum) verzettelt, desto mehr wird der Blick aufs Ganze, also auf relevantes bzw. wissenswertes Wissen, verwirrt.

Spätantik-frühmittelalterliche Summa: Eine dritte Spielart menippeischen Schreibens zeichnet sich nach Kirk (1980) in den dark ages des Niedergangs des römischen Weltreichs und des Aufstiegs des Christentums ab. Die Noctes Atticae des Gellius (2. Jh.), die Saturnalia des Macrobius (ca. um 400), Capellas De Nuptiis Philologiae et Mercurii (frühes 5. Jh.), Fulgentius' Mytologiarum Libri III (frühes 6. Jh.) und Boethius' De Consolatione Philosophiae (ca. um 525) gehören zu dieser Tradition. Im Mittelalter wird diese Tradition des summierenden Wissenskompendiums der vorchristlichen Antike vom Christentum und den Kirchenvätern 
aus christlicher Warte übernommen und kulminiert in der Summa Theologica des Thomas von Aquin (13. Jh.). Umberto Eco beschreibt die Methodik der vom Christentum absorbierten enzyklopädistischen Summa wie folgt:

The medieval thinker cannot conceive, explain, or manage the world without inserting it into the framework of an Order [...]. For the medieval thinker, the objects and events which the universe comprises are numerous. A key, therefore, must be found to help the scholar discover and catalogue them. The first approach to the reality of the universe was of an encyclopedic type. It was the first in the sense that the great popular encyclopedias, De Imagine Mundi, Specula Mundi, The Herbary or The Bestiary, historically preceded the epoch of the great theological arrangements. It was also the first in the sense that it was the most immediate, the most familiar and remains as a mental plan in even the most elaborate philosophical treatments. The encyclopedic approach uses the techniques of the Inventory, the List, the Catalogue or, in classical rhetorical terms, the Enumeratio. In order to describe a place or a fact, the early poets of the Latin Middle Ages first provide a list of detailed aspects.

(Eco 1982, 7-8)

Da obiger Textauszug aus Umberto Ecos The Aesthetics of Chaosmos. The Middle Ages of James Joyce stammt, ist der vorliegende Beitrag nun an jenem Dreh- und Angelpunkt angekommen, der die Theorie der menippeischen Prosaexegese mit der Lektürepraxis ausgewählter Textauszüge von Joyces Ulysses und Finnegans Wake verbindet.

\section{James Joyce und die Tradition menippeischen Schreibens}

Da im aufkommenden Mittelalter das Weltbild der Antike durch das des Christentums überformt wird, versuchen die an der menippeischen Summa schreibenden Enzyklopädisten, das Wissen der alten Welt zu archivieren. Hannu K. Rijkonen beschreibt dies wie folgt:

The encyclopaedic method used by the medieval scholars was typical of a civilisation which had to reconstruct a new world image on the ruins of the pagan Roman Empire but which had not yet any coherent view of the emerging new culture. [...] Joyce, for his part, tried to destroy the image of the world provided by the traditional culture.

(Rijkonen 1987, 39)

Da sich Joyce durch die am Jesuitenkolleg erworbene theologische Schulung paralysiert fühlt, arbeitet er sich sein ganzes Leben lang an der Überwindung des christlichen Weltbildes durch die Archäologie des Wissens der vorchristlichen Antike ab. Während er als angehender Jesuit in der Tradition der christianisierten Summa geschult wird, richtet Joyce den Blick auf deren menippeischen Widerpart, der das vorchristliche Weltbild der Antike zu archivieren sucht. Joyces 
Lebenswerk ist daher sowohl privat als auch künstlerisch ein zutiefst menippeisches Projekt, wenn man bedenkt, dass Julia Kristeva die Motivation menippeischen Schreibens in Rekurs auf Bakhtin als »struggle against Christianity and its representation « (Kristeva 1980, 80) bezeichnet.

Joyce schreibt sich jedoch nicht nur in die menippeische Tradition ein, um sein eigenes Denken durch eine Archäologie vorchristlichen Wissens von der Indoktrination des Christentums zu befreien. Es geht ihm wie zahlreichen Künstlern seiner Generation insbesondere auch um die abendländische Identitätskrise des großen Krieges in der eigenen Lebensgegenwart. Er nutzt die Tradition menippeischen Schreibens als Medium, um das kollektive Trauma, das das christliche Abendland durch den großen Krieg von 1914-1918 erfährt, aus dem Geist der vorchristlichen Antike heraus zu überwinden. Und auf diese Weise geht er als modernistischer Künstler (wie sein deutscher Kollege Thomas Mann, den er wohl nie richtig kennengelernt hat) einen eigenen, ganz besonderen Weg.

Während die literarische Avantgarde um Ezra Pound vor dem Ende des Ersten Weltkriegs unter dem Motto >make it new radikal in die Zukunft blickt und einen Bruch mit der althergebrachten Tradition anstrebt, revolutioniert James Joyce als High Modernist die Kunst im Dialog zwischen Tradition und Innovation. Um die durch den großen Krieg ausgelöste Identitätskrise der westlichen Welt zu bewältigen, transportiert er die homerischen Gründungsmythen unserer Kultur in die Gegenwart. T.S. Eliot bezeichnet dies als »mythical method«, bzw. als "a way of controlling, of ordering, of giving a shape and a significance to the immense panorama of futility and anarchy which is contemporary history« (1975 [1923], 177).

Indem er der kollektiven Identitätskrise der Gegenwart in Rekurs auf die homerischen Gründungsmythen neue Bedeutung und Kontinuität abringt und die heroisch-übermenschliche Welt des Mythos unter komischem bzw. mock-heroischem Vorzeichen auf normalmenschliches Maß herunterbricht, macht Joyce genau das, was die Menippea seit Jahrtausenden betreibt. Dies konkretisiert sich in Ulysses dadurch, dass jenes Narrativ den bereits erwähnten menippeischen Textbaustein der komischen Odysseusfigur aufgreift und dieser Figur in Kombination mit der ebenfalls als Konstante menippeischen Schreibens genannten sokratischen Philosophie Züge des Sokrates verleiht (vgl. Fuchs 2006, 123-129; 2009, 2011, 2017, 2020b).

Indem sich Joyce im Besonderen und die Menippea im Allgemeinen rückwärtsgewandt und selbstironisch bzw. selbstreflexiv auf den traditionellen literarischen Kanon - insbesondere Homers Gründungsmythen und die dort modellierte Odysseusfigur - beziehen, wird im Vorgriff auf eine Theorie der Prosa folgendes Phänomen erkennbar: Obschon die kanonische Weltsicht der homerischen Epen radikal als anarchisch wuchernde Antistruktur in Frage gestellt wird, leistet der intertextuelle Aufruf der homerischen Texte trotz ironischer Brechung nicht nur Arbeit am 
traditionellen kulturellen Gedächtnis, sondern fungiert auch als implizites Formund Strukturprinzip - als strukturierendes Muster, das den formalen Horizont bereitstellt, vor dessen Hintergrund autoreferentielle Metaisierung und experimentelle Deviation und Innovation wahrnehmbar gemacht werden können. Denn um eine Norm überschreiten zu können, muss diese zunächst durch Markierung kenntlich gemacht werden.

Ulysses: Als Teil der >mythical method ist Leopold Bloom als Hauptfigur von Ulysses nicht nur Widerpart des homerischen Odysseus, der im lateinischen Ulixes bzw. anglisiert Ulysses heißt. In Leopold Bloom treffen sich zudem die literarischen Bearbeitungen des homerischen Odysseus durch Dante, Shakespeare, Lord Tennyson usw. Und trotz dieses intertextuellen Überbaus hat der Protagonist Leopold Bloom keine Ahnung, dass die Ereignisse seines völlig unspektakulären Dubliner Alltags zentralen Episoden der homerischen Odyssee und ihrer künstlerischen Bearbeitung durch die bereits genannten Autoren entsprechen.

Aus den Gesprächen, die Joyce 1918 in Zürich mit seinem Freund Frank Budgen geführt hat, wissen wir, dass er Odysseus als Jedermann-Figur sieht, die nicht nur an die Gegenwart des großen Krieges andockbar ist, sondern auch in exemplarischer Weise die daraus resultierenden Traumata $\mathrm{zu}$ bewältigen versteht. Für Joyce spiegelt das Schicksal des Odysseus die Situation zahlreicher Veteranen der Lost Generation wider - junge Männer, die wie Odysseus von den Kriegstreibern manipuliert werden, ihre jungen Familien zu verlassen, viele Jahre hindurch in einen traumatisierenden Stellungskrieg gezwungen werden und trotz physischen Überlebens psychisch nicht mehr in der Lage sind, unbeschadet ins zivile Leben zurückzufinden und ans familiäre Glück vor dem Krieg anzuknüpfen (Budgen 1972, 17). Wie viele traumatisierte Veteranen des großen Krieges der Gegenwart schafft es Odysseus nicht, nach dem Kriegsende von Troja nach Hause zu fahren und dort für immer glücklich zu leben. Stattdessen irrt er zehn Jahre durch die Nachkriegswelt und als er es schafft, endlich nach Hause zu kommen, hat er sich so sehr verändert, dass ihn sogar die liebenswürdigste und treueste aller Ehefrauen der Weltliteratur zunächst nicht wiedererkennt. Indem er sich nicht auf die Geschichte des in der Ilias erzählten großen Krieges, sondern auf die Heimkehrversuche des Odysseus konzentriert, versucht Joyces Ulysses der orientierungslos gewordenen Gegenwart etwas Orientierung abzuringen.

Um zu skizzieren, wie sehr sich Joyce bei der Gestaltung seiner Odysseusfigur an der menippeischen Tradition orientiert, möchte ich exemplarisch auf zwei Aspekte verweisen, die anderswo in detaillierterer Weise nachlesbar sind.

Obwohl die zahlreichen Adaptationen des homerischen Odysseus in Joyces Ulysses intensiv erforscht sind, bedurfte es einer menippeischen Lektüre, um nachzuweisen, dass sich Joyce intertextuell auch auf die Parodie der Odysseusfigur in Lukians Nekyomantia bezieht. Wie die im Titel erwähnte Nekya vermu- 
ten lässt, erfolgt in diesem Text eine Parodie der Unterweltsreise des Odysseus durch die lukianische Figur des Menippos. Um die Unterwelt unbeschadet wieder verlassen zu können, tarnt sich der lukianische Menipp mit den Attributen dreier mythischer Figuren, von denen verbürgt ist, dass sie die Unterwelt besucht haben und es vermochten, von diesem lebensbedrohlichen Ort wieder unbeschadet zurückzukehren: Odysseus, Orpheus und Herakles. Bei Lukian trägt der unterweltsfahrende Menippos als Tarnung den Hut des Odysseus, die Lyra des Orpheus und das Löwenfell des Herakles. Genau mit diesen Attributen wird Leopold Bloom in der Circe-Episode des Ulysses versehen - ein Kapitel, das deutlich nachvollziehbar als menippeische Unterweltsfahrt konzipiert und wie Lukians Intertext in Dialogform als Binnendrama in die Prosa des Ulysses eingebettet ist (Fuchs 2006, 123-129; 2009, 2015).

Ebenso bedurfte es einer menippeisch orientierten Lektüre, um zu erkennen, dass nicht nur das Oxen of the Sun-Kapitel als Parodie von Platos Symposion konzipiert ist (Allison 1979), und Leopold Bloom dort nicht nur als komischer Odysseus, sondern auch als Parodie des platonischen Sokrates auftritt - eine menippeische Personalunion, die auch für die Makrostruktur des Ulysses rekonstruierbar ist und das Verständnis dieses Texts als Analogien- und Korrespondenzengeflecht revolutioniert (Fuchs 2011, 2015).

Finnegans Wake: Nach siebenjähriger Arbeit wurde 1922 Ulysses veröffentlicht. 1939 erschien Finnegans Wake, an dem Joyce ca. 16 Jahre lang gearbeitet hat. Hätte man mich vor Jahren gefragt, ob Finnegans Wake als Menippea konzipiert ist, hätte ich dies trotz einiger gegenteiliger Verdachtsmomente eher verneint.

Denn die Forschung tendiert dazu, jenen Text in großer Differenz zu allen traditionell überlieferten Prosa-Formen (einschließlich der ımythical method des Ulysses) zu lesen. Finnegans Wake ist zyklisch als narrative Endlosschleife konzipiert, hat keine klar erfassbaren Figuren, geschweige denn einen identifizierbaren Plot; wenn überhaupt greifbar, folgt letzterer einer Art Logik des Traumes, die alle Wahrnehmungskoordinaten unserer Wirklichkeitskonzeption ins Wanken bringt bzw. sogar auflöst, ähnlich einem Fiebertraum oder einer psychedelischen Grenzerfahrung. Daher, so die generelle Tendenz der Exegese, sei Finnegans Wake etwas völlig unerhört Neues und müsse auch als solches gedeutet werden.

Was mich aber hinsichtlich dieser Einschätzung nie völlig überzeugt hat, ist die Tatsache, dass Joyce in allen Texten, die er vor Finnegans Wake schrieb, Innovation aus der abendländischen Tradition literarischen Schreibens entbunden hat. Warum sollte dies in seinem letzten Werk überhaupt nicht mehr der Fall und völlig anders sein? Auch wenn Finnegans Wake den Joyce'schen Dialog mit der abendländischen Literatur- und Geistesgeschichte weiter radikalisiert, so scheint dieser Text dennoch auch durch jene Analogien- und Korrespondenzen- 
technik strukturiert, die als >mythical method für Ulysses und auch frühere JoyceTexte - beispielsweise The Dead (Fuchs 2020a) - nachgewiesen ist. Denn so heterogen der Wake-Text auf den ersten Blick scheint, so kann er dennoch wie Ulysses klar nachvollziehbar in Analogien- und Korrespondenzengeflechte eingeordnet werden, und dasselbe gilt auch für die aus allen Sprachen der Welt montierte, als Wakese bekannte Meta-Sprache des Textes. Im Folgenden gilt es daher, Finnegans Wake zunächst in linguistischer Hinsicht, dann in Rekurs auf das menippeisch durchbrochene Strukturgerüst der >mythical method $z u$ lesen und in exemplarischer Weise traditionelle Kompositions- und Strukturprinzipien zu rekonstruieren, vor deren Hintergrund die menippeisch-anarchisch-selbstreferentielle >Antistruktur` von Finnegans Wake als Deviation wahrnehmbar wird.

Die als Wakese bekannte Meta-Sprache des Textes wurde von Paul Fagan in einer Magisterarbeit erörtert, die laut gutachterlicher Stellungnahme seines Betreuers Werner Huber Dissertationsniveau aufweist und $\mathrm{zu}$ folgender Schlussfolgerung kommt:

[...] Joyce composed the Wake almost exclusively in neologisms, resulting in the work's infamy as a work of immeasurable difficulty, and even meaninglessness.

By interweaving linguistic and literary analyses, this study demonstrates that characterisations of the Wake's language as nonsensical, or as constituting a new language of $>$ Wakeser, result from the deficit of methodical explorations of the book's processes of signification. In order to correct this critical shortfall, this thesis shifts focus from what the text means to how it manages to do so, despite the odds. This exploration of the linguistic and semiotic processes of Finnegans Wake ultimately results in a surprising, even counterintuitive, demonstration that the code which invests the text with pragmatic significance is, in fact, the English linguistic code which Joyce, in writing his last work, is so often assumed to have abandoned.

(Fagan 2010, iv)

Indem Fagan zeigt, dass Wakese zwar Aspekte aller Sprachen absorbiert, dennoch aber lesbar ist, da es dem linguistischen Code des Englischen folgt, widerruft er die gängige Meinung, es handle sich dabei um eine notorisch unergründliche, strukturlose oder sogar widersinnige Sprache (Fagan 2010, v). Indem Joyce wie Rabelais Sprache wuchern und mäandern lässt und durch dieses Schlingern das strukturalistische Differenzprinzip teilweise suspendiert, zielt er nicht darauf ab, die Repräsentationskraft - also den linguistischen Code bzw. die >Grammatik « - dieses Zeichensystems auszuhebeln, sondern die konventionsgesteuerte Arbitrarität dieses Regelwerks als Metaebene fassbar zu machen. Wakese ist also Metasprache und keine unergründbare >Antispracheく.

Erweitert man nun den Blick von linguistischen auf literarische Strukturprinzipien wie die menippeisch gebrochene `mythical method mutung, dass Finnegans Wake nicht nur als nie dagewesener Antitext, sondern auch als Teil der traditionsgebundenen Joycekunst gelesen werden kann, durch 
eine Studie bestätigt, die Donald Philip Verene 2016 veröffentlicht hat: Joyce and the Philosophers at Finnegans Wake. Verene erbringt den überzeugenden Nachweis, dass Finnegans Wake analog zu Ulysses der >mythical method ‘ folgt. So wie die homerische Odyssee und deren menippeisches rewriting ein Strukturgerüst für Ulysses bilden, ist Finnegans Wake nach dem Modell von Vicos Principi di Scienza Nuova (1725) als Strukturgerüst modelliert. Diese Gemeinsamkeit zeigt sich insbesondere daran, wie Joyce diese Strukturgerüste für beide Texte nachträglich erläutert hat. Da die frühen Leserinnen sowohl Ulysses als auch Finnegans Wake aufgrund der Komplexität der Joyce’schen Anspielungshorizonte nicht verstehen konnten, hat der Autor rezeptionsfördernd strukturelle Erläuterungen nachgelegt. Diese erlangten im Fall Ulysses als von Joyce entworfene Korrespondenzenskizzen allgemeine Bekanntheit (vgl. Linati-Schema und Gordon Gilbert Plan), im Fall Finnegans Wake jedoch nicht. ${ }^{1}$

Nimmt man die von Joyce höchstpersönlich autorisierten Strukturierungshinweise in Rekurs auf Vico ernst, scheint es zudem relevant, dass Verene Vico als Sokratesfigur bezeichnet und somit die sokratische Erkenntniskritik als weiteren, für Finnegans Wake relevanten Textbaustein menippeischen Schreibens thematisiert:

[C]onsidering the circumstance that Vico fashioned himself as a new Socrates whose "science is the science of self-knowledge ([Verene 2016,] 79, 98) - Verene is certainly right to say that, as a rewriter of Vico, Joyce is a Socratic seeker of self-knowledge himself. As a key to the insight obtained when one is aware that one knows nothing, "Joyce's irony is Socratic irony« ([Verene 2016,] 96).

(Fuchs 2018, 188)

Diese sokratische, alles hinterfragende Dimension ist in Verknüpfung mit dem menippeischen Textbaustein der ironisch gebrochenen Odysseusfigur als gegen traditionelles Schreiben gerichtete Antistruktur von Finnegans Wake rekonstruierbar.

Bereits der Buchtitel Finnegans Wake scheint hinsichtlich der menippeischen Parodie der Odysseusfigur vielversprechend. Er ist prosimetrisch der bekannten irischen Ballade Lots of Fun at Finnegans Wake entnommen, die vom irischen Schluckspecht und Bauarbeiter Tim Finnegan handelt - eine strukturlose, kaum fassbare Anti-Persona, die in Joyces Finnegans Wake als Kneipenwirt Humphrey Chimpden Earwicker alias HCE oder Here Comes Everybody auftritt, jedoch ebenso durch ein Analogiengeflecht mit Ibsens Baumeister Solness, dem nursery rhyme >Humpty Dumpty` und den Freimaurern Strukturierung erfährt. In der Ballade scheint der Maurer Tim Finnegan einen Sturz von der Leiter mit seinem Leben zu bezahlen. Nach gut irischer Art versammelt sich die Nachwelt

1 Vgl. Ellmann 1982, 564: «He urged her [Harriet Shaw Weaver] now to read Vico's Scienza Nuova, as with Ulysses he had urged her to read the Odyssey." 
um den aufgebahrten Leichnam, um Tim Finnegan ins Totenreich zu geleiten, d. h., die Nacht vor seiner Beerdigung mit ihm in einer Whiskeyorgie zu verbringen. Dies ist das irische Ritual der als Wake bezeichneten karnevalesken Totenwache, das als christianisierte Unterweltsfahrt an den klassischen Archetypus der Nekya andockbar ist. Als einer der Trinkkumpanen etwas Whiskey verschüttet und damit den Leichnam benetzt, erwacht Tim Finnegan wieder zum Leben und feiert mit seinen Freunden bis in den Morgen hinein feuchtfröhliche Auferstehung. Da Whiskey auf Gälisch >Wasser des Lebens tion schlüssig; sie kann als menippeische Parodie eines Leben und geheimes Wissen spendenden Trinkrituals, also als Pseudoeucharistie, pseudo-platonisches Symposium oder als menippeische Unterweltsfahrt gedeutet werden. Als menippeische Parodie der klassischen Unterweltsfahrt fungiert daher die Totenwache in Finnegans Wake als irische Variante der Nekya des lukianischen Menippos, der diejenigen Hadesreisenden imitiert, denen es wie dem auferstehenden Tim Finnegan gelungen ist, nach dem Besuch der Unterwelt wieder in die Lebenswelt des Hier und Jetzt zurückzukehren: Herakles, Orpheus und Odysseus. Tim Finnegan wäre demzufolge wie der hadesreisende Menipp bei Lukian als menippeisch-komische Odysseus- bzw. Jedermannfigur zu verstehen, die den eigenen Abstieg ins Totenreich überlebt.

Verfolgt man diese archetypische Fährte weiter, so kann man neben der bereits skizzierten komisch gebrochenen Unterweltsfahrt einen weiteren epischen Strang rekonstruieren, der die menippeische Tradition der Parodie des homerischen Odysseus fortschreibt. In Finnegans Wake findet sich folgende Textzeile: »The house of Atreox is fallen indeedust (Ilyam, Ilyum! Maeromor Mournomates!) « ${ }^{2}$ Diese Textpassage eröffnet deutlich nachvollziehbar einen intertextuellen Dialog mit Ulysses als Paradebeispiel der von T.S. Eliot erörterten smythical method : »Fuit Ilium! The sack of windy Troy. Kingdoms of this world. The masters of the Mediterranean are fellaheen today.« (Joyce 1986, 7, 910-911) Die Begriffe »Maeromor « und "masters of the Mediterranean« sind unschwer auf Triest beziehbar: der Seehafen, von dem aus Österreich-Ungarn vor dem Ersten Weltkrieg das Mittelmeer kontrollierte. James Joyce hat in Triest über zehn Jahre gelebt und konnte vom Triestiner Hafen jederzeit die Silhouette der dortigen Habsburger-Residenz, Schloss Miramar alias »Maeromor«, sehen. Dieses Szenario bringen die zitierten Textauszüge mit dem Haus des Atreos (in das die schöne Helena vor ihrer Entfüh-

2 Joyce 1975, 55. Zitate aus dem Wake werden im Folgenden mit der Sigle FW und der Seitenzahl direkt im Fließtext angegeben. 
rung nach Troja einheiratet ${ }^{3}$ ) und dem Fall Trojas alias Ilium in Verbindung. Als geflügeltes Wort ist der Satz »Fuit Ilium« auf Vergils Aeneis (1930, II.325) als römisches Nachfolgeepos der homerischen Gründungsepen beziehbar.

Wie die Verweise auf den Fall Trojas und Schloss Miramar bzw. »Maeromor« bei Triest zeigen, gestaltet Joyce den Marine-Stützpunkt, den die Habsburger mit dem Fall des K\&K-Reichs Ende des Ersten Weltkriegs verlieren, als neues Troja. Auf diese Weise wird der Erste Weltkrieg mit dem Trojanischen Krieg und, wie bereits angedeutet, einer menippeischen Parodie der homerischen Odysseusfigur als Trojabesieger vernetzt. Dies erfolgt neben der analogen Überblendung des gefallenen Troja mit dem Haus Habsburg, das Schloss Miramar errichtete, dadurch, dass der Besitzer von Miramar - Erzherzog Ferdinand Maximilian von Habsburg, Bruder von Kaiser Franz Joseph - als unfreiwillig komische Odysseusfigur verstanden werden kann. Der Erzherzog fühlte sich von der See magisch angezogen, kommandierte die österreichisch-ungarische Marine und begab sich von 1857-1859 auf eine weltumsegelnde Expedition, die nach der Fregatte Novara als österreichisches Flaggschiff benannt ist und unter dem Deckmantel der Wissenschaft imperiale Handelsrouten erkundete. Letzterer Aspekt ist an die Joyce'sche Deutung der homerischen Odyssee anschließbar:

[Ulysses] didn't want to go off to Troy; he knew that the official reason for the war, the dissemination of the culture of Hellas, was only a pretext for the Greek merchants, who were seeking new markets.

(Ellmann 1992, 416)

Darüber hinaus fungiert Erzherzog Ferdinand Maximilian als unfreiwillige Parodie der Odysseusfigur in Dantes Divina Commedia. Bei Dante zieht es den alten Odysseus nach der Rückkehr in seine Heimat Ithaka nochmals zur See. Von unstillbarer Abenteuerlust getrieben, verlässt Dantes Odysseus das Mittelmeer alias »the Mediterranean [Sea]«, bricht in die terra incognita jenseits der Säulen des Herakles auf und bezahlt diesen Akt der Hybris mit dem Tod in den Weiten des Atlantiks. Analog hierzu bricht Erzherzog Ferdinand Maximilian von Miramar auf, um Kaiser von Mexiko zu werden, und schifft sich über das als Säulen des Herakles bekannte Gibraltar in die neue Welt ein. Als Akt der imperialen Selbstüberschätzung bezahlt er wie Dantes Odysseus die eigene Courage mit dem Leben: Nach bürgerkriegsähnlichen Unruhen wird der vermeintlich gottbegnadete Kaiser in Mexiko von einem Kriegsgericht zum Tode verurteilt und standrechtlich erschossen.

3 Da der Trojaner-Prinz Paris die Ehefrau Helena des Atridenfürsten Menelaos raubt, eröffnet dessen Bruder Agamemnon - der Anführer des Hauses der Atriden alias "[t]he house of Atreox «- den Trojanischen Feldzug. Obschon Troja durch die List des Odysseus fällt und das Haus der Atriden erfolgreich aus dem Krieg hervorgeht, richtet es sich im familiären Teufelskreis der Rache selbst zugrunde, daher die Wendung »The house of Atreox is fallen indeedust «. 
In Heinrich Vierordts Der Traum von Miramar - einem langen panegyrischen Gedicht - wird der auf der Fregatte Novara als Leichnam in die alte Welt zurück überführte Erzherzog als neuer Odysseus gefeiert. Da es sich dabei um ein miserables Werk eines nationalistischen Amateurdichters handelt, verliert das durch sein unrühmliches Ende vor dem Kriegsgericht bereits beschädigte Image des Erzherzogs zusätzlich an Fallhöhe:
[...]
Kein Freudenruf erfüllt die Luft,
Den Sarg birgt die Fregatte,
Daß Max man in der Väter Gruft
Daheim zu Wien bestatte ...
Am Meere wölbt sein Grabgehäus,
Wer dann es schaut, bewundert's;
Darin laßt ruhn den Odysseus
Des neunzehnten Jahrhunderts;

(Vierordt 1884)

Obwohl er sich als Odysseusfigur inszeniert, gleicht das Schicksal des kläglich scheiternden Erzherzogs dem des trojanischen Königs Priamos - ein Aspekt, der in Finnegans Wake durch die Zeichnung der Heimat des Erzherzogs, Schloss Miramar als neues Troja, unterstrichen wird. Während Odysseus durch eine List Troja erobert und zu Fall bringt, geht das als neues Troja gezeichnete Haus Habsburg am Ende des großen Krieges unter.

Als weiterer analog-strukturierender Anknüpfungspunkt für die zitierte Textpassage aus Finnegans Wake scheint es beachtenswert, dass Schloss Miramar genau dort erbaut wurde, wo der Vater des Odysseus zusammen mit dem Kynikerarchetypus Herakles von einer Unterweltsfahrt zurückgekehrt sein soll. Diese Unterweltsfahrt ist Teil des Argonautenmythos, der den Habsburgern über den Orden des Goldenen Vlieses (s. Grillparzers Dramen etc.) zur imperialen Selbstinszenierung dient. ${ }^{4}$ Nachdem die von Jason befehligten Argonauten zusammen mit dem Vater des Odysseus Troja bereits eine Generation vor der homerischen Ilias schon einmal besiegt haben sollen und nach einer odysseeähnlichen Irrfahrt in Colchis das Goldene Vlies erbeuten, kehren sie der Sage zufolge über einen acheronähnlichen Unterweltsfluss ins Mittelmeer zurück. Dabei handelt es sich um den Fluss Timavo, der wenige Kilometer von Schloss Miramar entfernt aus einer unterirdischen Höhle ins Meer mündet. Da sich in Schloss Miramar ein Gemälde von Cesare Dell'Acqua befindet, das die Rückkehr der Argonauten aus der Unterwelt ins Mittelmeer an der Costiera Triestina zeigt, wird der Argonauten-Mythos als pseudo-

4 Vgl. u. a. Leidinger 2003; Thanner 1992; Vocelka und Heller 1997. 
odysseischer Legitimationstext der Habsburg-Dynastie aufgerufen. Indem Miramar als imperiales Wohngebäude das Haus Habsburg metonymisch repräsentiert, wird dessen Herrschaftsanspruch gewissermaßen in Stein gemeißelt. Und dennoch ist sein Fall als neues Troja unvermeidlich: »The house of Atreox is fallen indeedust (Ilyam, Ilyum! Maeromor Mournomates!)« (FW, 55)

Wie die Lektüre einer einzigen Textzeile zeigt, ist Finnegans Wake aller experimentellen Radikalität zum Trotz nicht nur - aber eben auch - ähnlich wie Ulysses lesbar. ${ }^{5}$ Postuliert man das Strukturierungsprinzip der >mythical method lässt sich ein intertextuell dichtes Geflecht menippeisch-mythenparodistischer Referenzen identifizieren. Neben Anspielungen auf die homerischen Gründungsmythen finden sich Verweise auf die Argonautenfahrt mit Jason, Herakles und dem Vater des Odysseus als Vorgeschichte der homerischen Ilias und Odyssee, die mit dem Verweis auf den Fall Trojas in Vergils Aeneis (»Fuit Ilium«, 1930, II.325) als Nachfolgeepos der homerischen Odyssee und Dantes Odysseusbearbeitung in der Divina Commedia analog vernetzt und mit der menippeischen Tradition der parodistischen Inszenierung der Odysseusfigur verknüpft werden. Diese Beobachtungen geben Anlass zum folgenden Schlussausblick.

\section{Schlussausblick auf eine Theorie der Prosa}

Der vorliegende Beitrag hat den Versuch unternommen, die Textur von Finnegans Wake gegen den Strich zu lesen. Er verstößt damit bewusst gegen das Cliché gewordene Unverständlichkeitsprinzip, das diesem Text unterstellt zu werden pflegt, indem er von der Prämisse ausgeht, dass sich mimetisch-formale Strukturierungsprinzipien und selbstreferentiell-anarchistische, santistrukturellı-semantische Verdichtung nicht ausschließen, sondern gegenseitig bedingen. Dies scheint nicht nur in Finnegans Wake im Speziellen der Fall, sondern auch in der Tradition menippeischen Schreibens bzw. im Feld der Prosa im Allgemeinen. Menippeisches Schreiben als sokratisch-selbstreflexives Hinterfragen allgemein tradierter Wahrheiten, Philosophien und Denkmuster, experimentelles rewriting tradierter Schreibweisen und Gattungskonventionen und anarchisch-experimentelles smaking it new

5 Dieser double-bind von Struktur und Antistruktur wird im Fall Joyce durch folgende Problematik intensiviert: Joyce versucht, die christlich überformte antike Weltsicht als menippeische Summa zu rekonstruieren und sich vom System der christlichen Summa und dem Denken der Kirchenväter durch Auflistung von Analogien und Korrespondenzen zu befreien. Wie die Analogien- und Korrespondenzentechnik der `mythical method` jedoch zeigt, schreibt Joyce gegen die christliche Weltsicht zwar an, vermag es aber nicht, den Diskurs der Kirchenväter als Kognitionsmuster zu überwinden; vgl. Fuchs 2020a. 
(Pound) durch Verdichtung, Fragmentarisierung und sprachliche Verfremdung muss zwangsläufig vor der etablierten Folie jener Aspekte erfolgen, gegen die es sich abzuheben sucht. Und dies erfolgt in Rekurs auf den traditionellen Kanon abendländischen Schreibens, dessen Ausgangspunkt die beiden homerischen Gründungsmythen markieren, in denen bereits die Odysseusfigur als Außenseiter, Skeptiker, Lügner, Listenerfinder, proto-sokratischer wise fool etc. eine Schlüsselrolle der Normabweichung einnimmt. Wie Joyce immer wieder betont, fasziniert ihn - und damit auch die Tradition menippeischen Schreibens - die Tatsache, dass Odysseus anders ist als jede andere Figur der Weltliteratur (Budgen 1972, 16-17) und daher Deviation vor der Folie der Norm markiert und reflektiert. In dieser Hinsicht wäre die polytrop-selbstdistanzierte ${ }^{6}$ Odysseusfigur als archetypische Reflektorinstanz selbstreflexiv-experimentellen Schreibens begreifbar.

Trotz ihrer anarchischen >Gegenstruktur` hebt sich daher die Menippea nicht nur von traditionellen Formen und Strukturmustern ab, sondern markiert diese auch in selbstreflexiver Weise. Sie leistet daher zumindest indirekt - gewissermaBen als ductus obliquus (das Motto der Utopia von Thomas Morus) - Arbeit am kulturellen Gedächtnis, obschon sie dieses ironisch und radikal in Frage stellt und seriokomisch der Lächerlichkeit preisgibt.

Dieses Paradox scheint ein Aspekt, der auch der Theorie der Prosa zugrunde liegt. Experimentelle Prosa im Allgemeinen und Finnegans Wake im Besonderen sind nur deshalb in ihrer formsprengenden Innovation und Metaisierung als anarchische Antistruktur wahrnehmbar, da diese vor der formgebenden Folie traditioneller Strukturprinzipien als Deviation wahrnehmbar wird. Analog dazu, dass Chaos und Kosmos keine Gegensätze sind, sondern sich gegenseitig bedingen, sollten auch die Begriffe Struktur und Antistruktur nicht als Binäroppositionen, sondern als komplementäre Funktionen gedacht werden:

The world's beginning, Hesiod reported, began with chaos. The concept of chaos in the Theogony, then, does not signify »confusion« or »disorder.« Etymologically, it derives from the verb »chainein « which means »to gape« or »to yawn.« The »chaos« that Hesiod perceived is therefore to be understood as an opening, a gaping expanse, a spreading of a spatial kind which is bare of any form or contour or end. This is not a nothing (or nothing). Rather, it is an Ur-raum to be imagined strictly for the emergence of a possibility of space giving room for a »cosmos."

(Schabert und von Heyking 2020, 1)

Nimmt man letztere Überlegung ernst, so wäre das Dilemma, das David Lodge in The Novelist at the Crossroads (1971) beschreibt, keine strukturalistische Zwick-

6 Als vielgestaltig-wandlungsreiche bzw. auch wendungsreiche Figur schlüpft Odysseus in unterschiedlichste Identitäten, deckt sich mit ironisch-selbstdistanzierenden bzw. selbstreflektierenden Pseudonymen wie `Niemand`, denkt quer, täuscht, spielt verrückt etc. 
mühle, sondern ein kreativ-offener Möglichkeitsraum. Nach Lodge kann die postmoderne Prosa mit dem aus der Tradition erwachsenden Dilemma der Innovation nur auf zwei gegensätzliche Arten umgehen. Da der Modernismus Lodge zufolge alle Spielarten der experimentellen Innovation bereits ergründet hat, verbleibt für die Literatur der Gegenwart entweder die Option, auf Kosten der Form und Transparenz noch radikaler zu schreiben als die experimentellen Schriftsteller der Moderne, oder aber unter Beibehaltung der traditionellen Form und Lesbarkeit eines Textes, Versatzstücke der Tradition spielerisch zu zitieren, montieren und als textuelles Recycling neu und selbstreflexiv zu kontextualisieren.

Werner Wolf (1993) erachtet die beiden Ansätze, auf dieses Dilemma zu reagieren, als binär-oppositionelles Problem der Differenz. Finnegans Wake hingegen zeigt, dass es sich bei diesen Optionen, die Tradition weiterzuschreiben, nicht um Gegensätzlichkeiten handelt, sondern um (in jeweils unterschiedlicher Intensität und Konsequenz) in sich greifende Schreibprinzipien, die im bakhtinschen Sinne dialogisch miteinander in Bezug stehen, sich durch wechselseitige Interaktion relativieren, kommentieren etc. Hinsichtlich dieses Aspekts scheint Finnegans Wake exemplarisch, da dieser Text formgebende Struktur und formsprengend-selbstbezügliche Antistruktur in besonderer Konsequenz bzw. in besonders harter Fügung dialogisch aufeinander projiziert.

\section{Literaturverzeichnis}

Allison, June: A Literary Coincidence? Joyce and Plato. In: James Joyce Quarterly 16.3 (1979), 267-282.

Bakhtin, Mikhail: Problems of Dostoevsky's Poetics. Hrsg. v. Caryl Emerson. Manchester [1929/63] 1984, 109-121.

Budgen, Frank: James Joyce and the Making of »Ulysses« and Other Writings. London 1972 [1934].

Castrop, Helmut: Die varronische Satire in England 1660-1690. Studien zu Butler, Marvell und Dryden. Heidelberg 1983.

Dante, Alighieri: The Divine Comedy. Übers. v. C.H. Sisson. Oxford 2008.

Diogenes Laertius: Lives of Eminent Philosophers. Bd. II. Übers. v. R.D. Hicks. London, New York 1925.

Eco, Umberto: The Aesthetics of Chaosmos. The Middle Ages of James Joyce. Übers. v. Ellen Esrock. Tulsa 1982.

Eliot, T.S.: »Ulysses, Order, and Myth.«In: Selected Prose of T.S. Eliot. Hrsg. v. Frank Kermode. London 1975 [1923], 175-178.

Ellmann, Richard: James Joyce. New and Revised Edition. New York 1982.

Fagan, Paul: »nat language at any sinse of the world«. The Processes of Signification in James Joyce's Finnegans Wake. MA-Thesis Wien 2010.

Frye, Northrop: Anatomy of Criticism. Princeton 1957. 
Fuchs, Dieter: Joyce und Menippos: A Portrait of the Artist as an Old Dog. Würzburg 2006. Fuchs, Dieter: Joyce, Lucian, and Menippus: An Undiscovered Rewriting of the UlyssesArchetype. In: James Joyce Quarterly 47.1 (2009), 140-146.

Fuchs, Dieter: ১Poor Penelope. Penelope Rich،. Sir Philip Sidney’s Astrophil and Stella as a Source for the Rewriting of the Odysseus-Archetype in James Joyce's Ulysses. In: James Joyce Quarterly 48.2 (2011), 350-356.

Fuchs, Dieter: ^He puts Bohemia on the seacoast and makes Ulysses quote Aristotler. Shakespearean Gaps and the Early Modern Method of Analogy and Correspondence in James Joyce's Ulysses. In: Joyce/Shakespeare. Hrsg. v. Laura Pelaschiar. Syracuse 2015, 21-37.

Fuchs, Dieter: James Joyce's Ulysses - A Menippean Satire? In: Joyce Studies in Italy 19 (2017), 79-90.

Fuchs, Dieter: Review: Donald Phillip Verene: James Joyce and the Philosophers at Finnegans Wake. Evanston 2016. In: James Joyce Quarterly 56.1 (2018), 185-189.

Fuchs, Dieter: James Joyce's The Dead and Macrobius's Saturnalia: The Menippean Encyclopedic Tradition and the Mythical Method. In: James Joyce Quarterly 57.3-4 (2020a), 275-292.

Fuchs, Dieter: Making History in Washington Irving's Rip van Winkle and James Joyce's Ulysses. In: Wherefrom Does History Emerge? Inquiries in Political Cosmogony. Hrsg. v. Tilo Schabert und John von Heyking. Berlin, Boston 2020b, 7-37.

Helm, Rudolf: Lucian und Menipp. Hildesheim 1967 [1906].

Joyce, James: Finnegans Wake. London 1975.

Joyce, James: Ulysses. Hrsg. v. Hans Walter Gabler. New York 1986.

Kirk, Eugene: Menippean Satire: An Annotated Catalogue of Texts and Criticism. New York 1980.

Koppenfels, Werner von: Mundus alter et idem. Utopiefiktion und menippeische Satire. Poetica 13 (1981), 16-66.

Kristeva, Julia: Word, Dialogue, and Novel. In: Desire in Language. A Semiotic Approach to Literature and Art. Hrsg. v. Leon S. Roudiez. Oxford 1980, 64-91.

Leidinger, Hannes: Der Mythos Habsburg. Eine Einleitung. In: Schwarzbuch der Habsburger. Die unrühmliche Geschichte eines Herrscherhauses. Hrsg. v. Hannes Leidinger, Verena Moritz und Berndt Schippler. Wien 2003, 2-14.

Lodge, David: The Novelist at the Crossroads and Other Essays on Fiction and Criticism. London 1971.

Quintilianus, M. Fabius: Institutionis oratoriae Libri duodecim. Hrsg. v. Michael Winterbottom. Oxford 1970.

Relihan, Joel C.: Menippus the Cynic in the Greek Anthology. In: Syllecta Classica 1 (1989), 55-61.

Relihan, Joel C.: Rethinking the History of the Literary Symposium. In: Illinois Classical Studies 17.2 (1992), 213-244.

Relihan, Joel C.: Ancient Menippean Satire. Baltimore 1993.

Rijkonen, Hannu K.: Menippean Satire as a Literary Genre with special Reference to Seneca's Apocolocyntosis. Helsinki 1987.

Schabert, Tilo und John von Heyking: Introduction. In: Wherefrom Does History Emerge? Inquiries in Political Cosmogony. Hrsg. v. Tilo Schabert und John von Heyking. Berlin, Boston 2020, 1-6. 
Simon, Ralf: Die Idee der Prosa. Zur Ästhetikgeschichte von Baumgarten bis Hegel mit einem Schwerpunkt bei Jean Paul. München 2013.

Simon, Ralf: Vor und nach der Form. Zur Temporalität des ästhetischen Formprozesses. In: Zeiten der Form - Formen der Zeit. Hrsg. v. Michael Gamper, Eva Geulen, Johannes Grave, Andreas Langenohl, Ralf Simon und Sabine Zubarik. Hannover 2016, 63-82.

Tanner, Marie: The Last Descendants of Aeneas. The Hapsburgs and the Mythic Image of the Emperor. New Haven 1992.

Verene, Donald Phillip: James Joyce and the Philosophers at Finnegans Wake. Evanston 2016.

Vierordt, Heinrich: Der Traum von Miramar [1884]. 29. Juli 2020: http://gedichte.xbib.de/Vier ordt_gedicht_Der+Traum+von+Miramar+-+III..htm.

Vergilius, Maro Publius: The Aeneid. Hrsg. v. J.W. Mackail. Oxford 1930.

Vocelka, Karl und Lynne Heller: Die Lebenswelt der Habsburger. Kultur- und Mentalitätsgeschichte einer Familie. Graz 1997.

Weinbrot, Howard D.: Menippean Satire Reconsidered: From Antiquity to the Eighteenth Century. Baltimore 2005.

Wolf, Werner: Ästhetische Illusion und Illusionsdurchbrechung in der Erzählkunst. Theorie und Geschichte mit Schwerpunkt auf englischem illusionsstörenden Erzählen. Tübingen 1993. 
